\title{
Thrombotic Thrombocytopenic Purpura Complicating Severe Legionnaires' Disease
}

Hammer $L^{1}$, Minet $C^{1}$, Bonadona $A^{1}$, Remy $J^{1}$, Maurin $\mathbf{M}^{2}$, Ara-Somohano $\mathbf{C}^{1}$, Hamidfar-Roy $\mathbf{R}^{1}$, Schwebel $\mathbf{C}^{1}$ and Timsit $\mathrm{JF}^{1^{*}}$

${ }^{1}$ University Grenoble, Teaching Hospital Albert Michallon, Medical Polyvalent ICU, Paris, France

${ }^{2}$ University Grenoble, Teaching Hospital Albert Michallon, Microbiology Department, Paris, France

*Corresponding author: Jean-Francois Timsit, University Paris Diderot - Bichat Hospital - Medical and Infectious Diseases ICU, Paris, Tel: +33140257702; Fax: +33140258837; E-mail: jftimsit@chu-grenoble.fr

Rec Date: Nov 03, 2013, Acc Date: May 30, 2014, Pub Date: June 3, 2014

Copyright: ( 2014 Hammer L, et al. This is an open-access article distributed under the terms of the Creative Commons Attribution License, which permits unrestricted use, distribution, and reproduction in any medium, provided the original author and source are credited.

\begin{abstract}
We describe the first case of thrombotic thrombocytopenic purpura (TTP) in a patient with Legionnaires' disease. TTP was demonstrated by the low level of adams TS13 activity that resolved after plasmapheresis and remained normal in the long-term follow up.
\end{abstract}

Keywords: Thrombotic thrombocytopenic purpura; Legionnaires' disease; Legionellosis; Legionella pneumophila; Plasmapheresis

\section{Case Report}

A 40-year-old male was admitted to the medical intensive care unit with acute respiratory failure. The patient had no medical history and smoked 10 cigarettes daily 10 years ago. One week before admission, the patient saw his primary care physician because of chest congestion, nonproductive cough and generalized myalgias. Symptomatic treatment with analgesic and antipyretic was administered. The day before admission his symptoms worsened with productive cough and severe shortness of breath. A chest radiograph was performed, which showed diffuse bilateral opacities. Treatment with ceftriaxone and betamethasone was given. Twelve hours later, the patient was admitted in intensive care unit due to severe shortness of breath. Upon arrival, he was conscious and afebrile, the blood pressure was $77 / 51 \mathrm{mmHg}$, pulse rate was $140 / \mathrm{min}$, and respiratory rate was $44 / \mathrm{min}$. He was severely hypoxemic with $50 \%$ oxygen saturation while breathing ambient air and his oxygen saturation dropped to 76 percent while breathing 100 percent oxygen with a face mask. He was promptly intubated and placed on mechanical ventilation. Cardiac examination revealed a regular rate and rhythm with no murmur, rubs or gallop, and lung examination revealed bilateral rales. The remainder of the physical examination was unremarkable. He was sedated with midazolam and sufentanyl, and paralyzed with cisatracurium. $\mathrm{He}$ received immediately vasopressor treatment with norepinephrin after fluid resuscitation. Antibiotherapy was started with levofloxacin, erythromycin and ceftriaxone. Hematologic and blood chemical values are given in Table 1 and Figure 1, respectively. The arterial lactates concentration was $8.5 \mathrm{mmol} / \mathrm{L}$. A chest radiograph showed progressive worsening of the opacities in both lungs. An electrocardiogram showed no abnormalities; neither did a transthoracic echocardiogram. Bronchoscopy and bonchoalveolar lavage were not performed immediately because of the hypoxemia. A sputum specimen contained few neutrophils with no microorganisms. Culture was started. A specific urinary-antigen test for detection of Legionella pneumophila serogroup 1 (Binax NOW Legionella ${ }^{\circ}$, Alere, 78350 Jouy-en-Josas, France) was positive. Therefore, he was diagnosed with adult respiratory distress syndrome and septic shock complicating Legionnaires' disease. The patient remained severely hypoxemic and needed high level of $\mathrm{FiO}_{2}$, peep and prone positioning for 5 days.

\begin{tabular}{|l|l|l|l|l|l|l|l|}
\hline Variable & On admission & Day 2 & Day $\mathbf{6}$ & Day $\mathbf{1 2}$ & Day $\mathbf{1 7}$ & Day 24 & Last ICU day \\
\hline Hematocrit (\%) & 45 & 35 & 19 & 24 & 25 & 25 & 29 \\
\hline $\begin{array}{l}\text { White-cell count } \\
\left(\text { per } \mathrm{mm}^{3}\right)\end{array}$ & 12,500 & 20,300 & 43,800 & 12,300 & 8300 & 8600 & 6900 \\
\hline Platelets (per mm $\left.{ }^{3}\right)$ & 15,000 & 62,000 & 27,000 & 184,000 & 28,000 & 312,000 & 459,000 \\
\hline Hemoglobin (g/L) & 164 & 123 & 72 & 83 & 69 & 86 & 100 \\
\hline Schistocytes (\%) & & & 10 & 1.6 & 5 & 0,2 & \\
\hline Creatinine (mg/dL) & 166 & 140 & 128 & 104 & 100 & 61 & 76 \\
\hline Sodium (mmol/liter) & 130 & 133 & 147 & 142 & 140 & 140 & 139 \\
\hline
\end{tabular}




\begin{tabular}{|l|l|l|l|l|l|l|l|}
\hline Haptoglobin (g/L) & & & 0.1 & 0.2 & 0.1 & 1.6 & \\
\hline ASAT (IU/L) & 242 & 648 & 155 & 75 & 53 & 25 & 36 \\
\hline ALAT (IU/L) & 64 & 168 & 64 & 51 & 46 & 17 & 56 \\
\hline LDH (IU/L) & & 5527 & 4608 & 918 & 1902 & 660 & \\
\hline
\end{tabular}

Table 1: Laboratory values

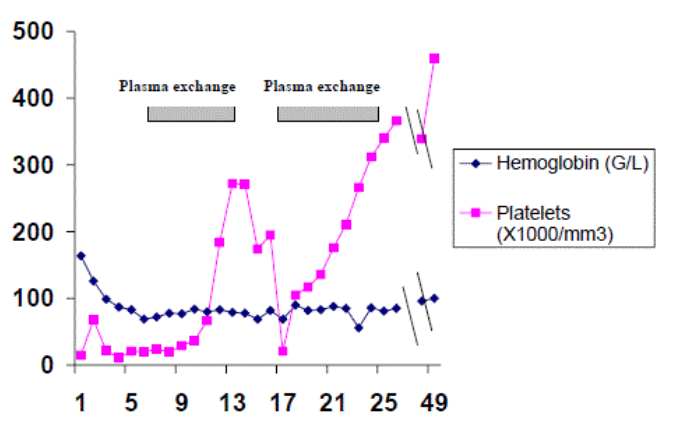

Figure 1: Plasma exchange courses and levels of platelet and hemoglobin over time.

On the second day, sputum culture showed several colonies of L. pneumophila serogroup 1. Ceftriaxone was stopped.

On the sixth day, the platelet count decreased to $20 \times 10^{3} / \mathrm{mm}^{3}$. A peripheral smear revealed microangiopathic hemolytic anemia with $10 \%$ schistocytes, and hemoglobin $72 \mathrm{~g} / \mathrm{L}$. The serum biochemistry showed $\mathrm{LDH}$ at $2589 \mathrm{UI} / \mathrm{L}$, haptoglobin at $0.1 \mathrm{~g} / \mathrm{L}$, impaired renal function with BUN at $17.7 \mathrm{mmol} / \mathrm{L}$ and creatinine at $128 \mu \mathrm{mol} / \mathrm{L}$. The prothrombin time, activated cephalin time, fibrinogen and d-dimmer were normal, and Coombs' test was negative. We measured Von Willebrand factor cleaving protease (ADAMTS-13) activity $<5 \%$ of normal. We found $57.2 \mathrm{U} / \mathrm{mL}$ inhibitor of ADAMTS-13. On the basis of the laboratory findings, thrombotic thrombocytopenic purpura (TTP) was diagnosed. Hepatitis panel and HIV serology were negative. Test for antinuclear antibodies and antineutrophilic cytoplasmic antibodies were negative. The results of serum complement testing and serum protein electrophoresis were also normal. A bone marrow aspirate revealed a hypercellular bone marrow with marked erythroid hyperplasia and megakaryocytic hyperplasia.

The patient began daily plasmapheresis with 1.5 plasma volumes of fresh frozen plasma. He had a good laboratory response after 4 sessions and his platelet count was $184 \times 10^{3} / \mathrm{mm}^{3}$. Plasma exchanges were stopped but thrombopenia reappeared with a platelet count of $28 \times 10^{3} / \mathrm{mm}^{3}$ and schistocytes increase of $5 \%$. We started new plasma exchanges daily during 5 days. We waited for 2 days after the platelet count returned to normal to stop plasmapheresis.

After 12 days of treatment, the patient showed signs of respiratory improvement with fraction of inspired oxygen needs of $40 \%$ on a positive end-expiratory pressure of $8 \mathrm{~cm} \mathrm{H}_{2} \mathrm{O}$. Sedation was discontinued but a tracheotomy was performed on the sixteenth day because of difficulties of weaning due to neurologic weakness, and deglutition disorders. Indeed, the patient was severely weak and a neurologic consultation was requested. On neurologic examination, he was drowsy and had poor orientation, attention, information, and recall. An EEG showed no abnormalities. A head MRI with and without gadolinium contrast showed several occipital ischemic lesions, likely due to initial low cardiac output.

Finally the patient's respiratory status slowly improved and mechanical ventilation was stopped one month after admission. His mental status also improved and he became fully oriented and deglutition disorders disappeared. Tracheotomy cannula was removed and he tolerated 3 to $4 \mathrm{~L}$ of oxygen by nasal cannula 45 days after admission.

The patient was finally discharged to a rehabilitation facility 50 days after admission. The ADAMS 13 levels returned to normal values. One year later, the patient had no relapse of TTP and remained in complete remission.

\section{Discussion}

We described here a patient with Legionnaire's disease in whom the diagnosis of secondary TTP was made on the basis of laboratory typical features. Classically TTP was diagnosed as a pentad of signs: microangiopathic hemolytic anemia, thrombocytopenia, acute renal failure, neurological abnormalities and fever. In this case the diagnosis was difficult, as the patient was sedated and neurological examination could not be performed. Thrombocytopenia has a high incidence in medical critically ill patients (about 40\%) [1,2]. Evaluation of microangiopathic hemolysis by determination of the schistocytes rate is not routinely performed.

Although infectious diseases have been considered as a contributing factor of TTP, the predisposing pathogens have rarely been reported in previous literature. In the Oklahoma Thrombotic Thrombocytopenic Purpura- Hemolytic Uremic Syndrom (TTP-HUS) Registry, 7\% of patients with an initial diagnosis of idiopathic TTP were subsequently found to have sepsis. Bacterial sepsis with Staphylococcus aureus, Streptococcus sp., Pseudomonas aeruginosa, Enterobacter sp., Rickettsia rickettsii were the most frequently reported. Other microorganisms may be occasionally involved such as Chlamydia pneumonia, fungi such as Pneumocystis jiroveci and viruses [3-5]. Many drugs have been reported as associated with TTP, such as quinine, ticlopidin, clopidogrel, oncological chemotherapeutic agents, and immunosuppressive agents. None of our patient's medications have been described as a cause of TTP. TTP associated with Legionnaires' disease has been suspected in only one case [6]. Legionnaires' disease or legionellosis is classically a severe pneumonia occurring after inhalation of aerosols formed from water contaminated with a Legionella species [7]. These Gram-negative bacteria are found in various aquatic environments, including in manmade water systems. Although more than 50 species have been characterized in the genus Legionella, L. pneumophila (especially its 
Citation: Hammer L, Minet C, Bonadona A, Remy J, Maurin M, et al. (2014) Thrombotic Thrombocytopenic Purpura Complicating Severe Legionnaires' Disease. J Med Microb Diagn 3: 138. doi:10.4172/2161-0703.1000138

Page 3 of 3

serogroup 1) is responsible for more than $90 \%$ of human infections [7]. The clinical symptoms of legionellosis pneumonia are poorly specific [7], and thus diagnosis is most often confirmed by detection of specific antigens in the urine of the infected patient. However, currently available urinary antigen tests only detect antigens from $L$. pneumophila sg 1 [8]. PCR-based tests have been recently introduced for diagnosis of legionellosis, and potentially allow detection of all Legionella spp. The mortality rate associated with legionellosis is on average $10-15 \%$, but may be much higher in immunocompromised patients [7]. Life-threatening complications include respiratory failure, septic shock, and acute renal failure, to which TTP should now be added.

The spontaneous mortality (without treatment) of TTP was initially close to $90 \%$, but plasmapheresis treatment has recently decreased it to $10 \%$. Indeed, for adults, plasma exchange is the only treatment considered as effective [9]. Its effectiveness has been attributed to the removal of ADAMTS-13 autoantibodies and replacement of ADAMTS-13 activity [10]. Daily plasma exchange is recommended with replacement of 1.0 to 1.5 times the predicted plasma volume of the patient [11]. The British guidelines recommend that plasmaexchange therapy be continued for a minimum of two days after the platelet count returns to normal level $\left(>150,000 / \mathrm{mm}^{3}\right)$ [11]. In our case, plasma exchange was stopped only one day after normalization of the platelet count, which may explain TTP relapse.

In conclusion, the present case demonstrates that acute, singleepisode of TTP can complicate Legionnaires' disease. Early recognition of the diagnosis and prompt treatment with plasma exchange and antibiotic therapy could achieve good clinical outcome.

\section{References}

1. Vanderschueren S, De Weerdt A, Malbrain M, Vankersschaever D, Frans E, et al. (2000) Thrombocytopenia and prognosis in intensive care. Crit Care Med 28: 1871-1876.
2. Crowther MA, Cook DJ, Meade MO, Griffith LE, Guyatt GH, et al. (2005) Thrombocytopenia in medical-surgical critically ill patients: prevalence, incidence, and risk factors. J Crit Care 20: 348-353.

3. George JN, Vesely SK, Terrell DR (2004) The Oklahoma Thrombotic Thrombocytopenic Purpura-Hemolytic Uremic Syndrome (TTP-HUS) Registry: a community perspective of patients with clinically diagnosed TTP-HUS. Seminars in hematology 41: 60-67.

4. Knox-Macaulay HH, Adil SN, Ahmed EM (2004) Acute thrombotic thrombocytopenic purpura following doxycycline treatment of Chlamydia pneumoniae infection in a patient with dermatomyositis. Clinical and laboratory haematology 26: 147-151.

5. Li CH, Ho CL (2007) Thrombotic thrombocytopenic purpura as the initial presentation of Pneumocystis jiroveci pneumonia in an immunocompetent patient. Ann Hematol 86: 223-224.

6. Riggs SA, Wray NP, Waddell CC, Rossen RD, Gyorkey F (1982) Thrombotic thrombocytopenic purpura complicating Legionnaires' disease. Arch Intern Med 142: 2275-2280.

7. Carratalà J, Garcia-Vidal C (2010) An update on Legionella. Curr Opin Infect Dis 23: 152-157.

8. Bartlett JG (2011) Diagnostic tests for agents of community-acquired pneumonia. Clin Infect Dis 52 Suppl 4: S296-304.

9. Rock GA, Shumak KH, Buskard NA, Blanchette VS, Kelton JG, et al. (1991) Comparison of plasma exchange with plasma infusion in the treatment of thrombotic thrombocytopenic purpura. Canadian Apheresis Study Group. N Engl J Med 325: 393-397.

10. Moake JL (2002) Thrombotic microangiopathies. N Engl J Med 347: 589-600.

11. Allford SL, Hunt BJ, Rose P, Machin SJ; Haemostasis and Thrombosis Task Force, British Committee for Standards in Haematology (2003) Guidelines on the diagnosis and management of the thrombotic microangiopathic haemolytic anaemias. Br J Haematol 120: 556-573. 